\title{
Article
}

\section{An Ideal-Based Dot Total Graph of a Commutative Ring}

\author{
Mohammad Ashraf ${ }^{1}$, Jaber H. Asalool ${ }^{1}\left(\mathbb{D}\right.$, Abdulaziz M. Alanazi ${ }^{2, *} \mathbb{(}$ and Ahmed Alamer ${ }^{2}(\mathbb{D}$ \\ 1 Department of Mathematics, Aligarh Muslim University, Aligarh 202002, India; \\ mashraf80@hotmail.com (M.A.); asalooljaber@gmail.com (J.H.A.) \\ 2 Department of Mathematics, University of Tabuk, Tabuk 71491, Saudi Arabia; aalamer@ut.edu.sa \\ * Correspondence: am.alenezi@ut.edu.sa
}

check for updates

Citation: Ashraf, M.; Asalool, J.H.;

Alanazi, A.M.; Alamer, A. An Ideal-Based Dot Total Graph of a Commutative Ring. Mathematics 2021, 9, 3072. https://doi.org/10.3390/ math9233072

Academic Editors: Irina Cristea and Hashem Bordbar

Received: 6 November 2021

Accepted: 25 November 2021

Published: 29 November 2021

Publisher's Note: MDPI stays neutral with regard to jurisdictional claims in published maps and institutional affiliations.

\begin{abstract}
In this paper, we introduce and investigate an ideal-based dot total graph of commutative ring $\mathcal{R}$ with nonzero unity. We show that this graph is connected and has a small diameter of at most two. Furthermore, its vertex set is divided into three disjoint subsets of $\mathcal{R}$. After that, connectivity, clique number, and girth have also been studied. Finally, we determine the cases when it is Eulerian, Hamiltonian, and contains a Eulerian trail.
\end{abstract}

Keywords: commutative rings; zero-divisor graph; dot total graph; ideal-based; zero-divisors

MSC: 97K30; 68R10; 94C15; 13A15

\section{Introduction}

Let $Z(\mathcal{R})$ and $\operatorname{Reg}(\mathcal{R})$ be a set of zero-divisors and a set of regular elements of commutative ring $\mathcal{R}$ with $1 \neq 0$, respectively. In [1], Mohammad Ashraf et al. defined the dot total graph of $\mathcal{R}$, denoted by $T_{Z(\mathcal{R})}(\Gamma(\mathcal{R})$ ), as an (undirected) graph, which consists of all elements of $\mathcal{R}$ as vertex set $V\left(T_{Z(\mathcal{R})}(\Gamma(\mathcal{R}))\right)$ and includes all edges such that for distinct $x, y \in \mathcal{R}, e=x y \in E\left(T_{Z(\mathcal{R})}(\Gamma(\mathcal{R}))\right)$ if and only if $x y \in Z(\mathcal{R})$. In this paper, we replace $Z(\mathcal{R})$ by an ideal $I$, and we introduce and investigate an ideal-based dot total graph of $\mathcal{R}$ denoted $T_{I}(\Gamma(\mathcal{R}))$. In addition, Redmond [2] defined $\Gamma_{I}(\mathcal{R})$ as an undirected graph. It has vertices $\{x \in \mathcal{R} \backslash I \mid x y \in I$ for some $y \in \mathcal{R} \backslash I\}$. In this case, $x$ and $y$ are vertices that are both distinct and adjacent if and only if $x y \in I$, i.e., $\Gamma_{I}(\mathcal{R})$ is subgraph of $T_{I}(\Gamma(\mathcal{R}))$. It will also appear in this paper. Further, if $I=(0)$ in $\Gamma_{I}(\mathcal{R})$, then $\Gamma_{I}(\mathcal{R})=\Gamma(\mathcal{R})$; this graph is studied by Anderson et al. [3], and they were interested in studying the interplay of ring-theoretic properties of $\mathcal{R}$ with graph-theoretic properties of $\Gamma(\mathcal{R})$. Moreover, they associated a (simple) graph $\Gamma(\mathcal{R})$ to $\mathcal{R}$, which consists of a vertex set $V(\Gamma(\mathcal{R}))=Z(\mathcal{R})^{*}=$ $Z(\mathcal{R}) \backslash\{0\}$ and edge set $E(\Gamma(\mathcal{R}))$ such that for all distinct $x, y \in Z(\mathcal{R})^{*}, e=x y \in E(\Gamma(\mathcal{R}))$ if and only if $x y=0$. Furthermore, if $I=(0)$ in $T_{I}(\Gamma(\mathcal{R}))$, then $T_{I}(\Gamma(\mathcal{R}))=\Gamma_{0}(\mathcal{R})$; this graph is studied by Beck [4], in which he considered $\mathcal{R}$ as a simple graph for which its vertex set is the set of all elements of $\mathcal{R}$ and edge set such that for all distinct $x, y \in \mathcal{R}$, $e=x y \in E\left(\Gamma_{0}(\mathcal{R})\right)$ if and only if $x y=0$. In addition, some fundamentals of Laplacian eigenvalues and energy of graphs can be identified in [5-7].

Assuming $G$ to be a graph, $G$ can be said to be connected when a path connects every pair of its distinctive vertices. Denoting distinct vertices of graph $G$ to be $x$ and $y, d(x, y)$ will indicate the shortest distance between the two vertices. However, where no such path exists, it will be represented by $d(x, y)=\infty$. Similarly, the diameter of $G$ is $\operatorname{diam}(G)=\sup \{d(x, y) \mid x$ and $y$ are distinct vertices of $G\}$. The girth of $G$, denoted by $\operatorname{gr}(G)$, is defined as the length of shortest cycle in $G(g r(G)=\infty$ if $G$ contains no cycle). Note that if $G$ contains a cycle, then $\operatorname{gr}(G) \leq 2 \operatorname{diam}(G)+1$. The degree of vertex $v$, written $\operatorname{deg}_{G}(v)$ or $\operatorname{deg}(v)$, is the number of edges incident to $v$ (or the degree of the vertex $v$ is the number of vertices adjacent to $v$ ). In a connected graph $G$, a vertex $v$ is said to be a cut-vertex of $G$ if and only if $G \backslash\{v\}$ is disconnected. Let $V(G)$ be a vertex set of $G$. Then, the subset $U \subseteq V(G)$ is called a vertex-cut if $G \backslash U$ is disconnected. The connectivity of a 
graph $G$ denoted by $k(G)$ and is defined as the cardinality of a minimum vertex-cut of $G$, which is also the same concepts we have in the edges. In a connected graph $G$, an edge $e$ is said to be a bridge of $G$ if and only if $G \backslash\{e\}$ is disconnected. Let $E(G)$ be an edge set of $G$. If $G \backslash X$ is disconnected, it will have a subset $X \subseteq E(G)$ as its edge-cut. Let $\lambda(G)$ denote the edge-connectivity of a connected graph $G$ which is the size of the smallest set of edges for which removal disconnects $G$. Moreover, a clique is a complete subgraph of a graph $G$. The clique number denoted by $\omega(G)$ is the greatest integer $n \geqslant 1$ such that $K_{n} \subseteq G$, and $\omega(G)=\infty$ if $K_{n} \subseteq G$ for all $n \geqslant 1$. A nontrivial connected graph $G$ is Eulerian if every vertex of $G$ has an even degree. Moreover, $G$ contains a Eulerian trail if exactly two vertices of $G$ have an odd degree. In addition, let $G$ be a graph of order $n \geq 3$. If $\operatorname{deg}(u)+\operatorname{deg}(v) \geq n$ for each pair $u$ and $v$ of vertices of $G$ that are not adjacent, then $G$ is Hamiltonian.

The present paper is organized as follows:

In Section 2, we define an ideal-based dot total graph of $\mathcal{R}$ and study the most basic results of $T_{I}(\Gamma(\mathcal{R}))$. We provide many examples and show that $T_{I}(\Gamma(\mathcal{R}))$ is always connected with $\operatorname{diam}\left(T_{I}(\Gamma(\mathcal{R}))\right) \leqslant 2$ and $g r\left(T_{I}(\Gamma(\mathcal{R}))\right) \leqslant 5$, and we determine when $T_{I}(\Gamma(\mathcal{R}))$ is a complete graph and a regular graph. Moreover, we find the degree of each vertex of $T_{I}(\Gamma(\mathcal{R}))$. Furthermore, in Section 3, we study the connectivity of $T_{I}(\Gamma(\mathcal{R}))$ when $T_{I}(\Gamma(\mathcal{R}))$ has a no cut-vertex, and $T_{I}(\Gamma(\mathcal{R}))$ has a bridge. We shall also find the $k\left(T_{I}(\Gamma(\mathcal{R}))\right)$. On the other hand, in Section 4, we study the clique number and girth of $T_{I}(\Gamma(\mathcal{R}))$, and we determine the clique number when $T_{I}(\Gamma(\mathcal{R}))$ has a cycle. Furthermore, we find the girth of $T_{I}(\Gamma(\mathcal{R}))$, i.e., $\operatorname{gr}\left(T_{I}(\Gamma(\mathcal{R}))\right)$. Finally, in Section 5, we study the traversability of $T_{I}(\Gamma(\mathcal{R}))$ when $T_{I}(\Gamma(\mathcal{R}))$ is Eulerian or contains a Eulerian trail, and $T_{I}(\Gamma(\mathcal{R}))$ is Hamiltonian.

\section{Definition and Basic Structure of $T_{I}(\Gamma(\mathcal{R}))$}

In this section, we define an ideal-based dot total graph, denoted by $T_{I}(\Gamma(\mathcal{R}))$, and show that this graph is always connected and has a small diameter of at most two. By dividing the element of $\mathcal{R}$ into three disjoint subsets, we study the basic results on the structure of this graph and the relationship between $T_{I}(\Gamma(\mathcal{R}))$ and $T_{Z(\mathcal{R})}(\Gamma(\mathcal{R})), T_{Z(\mathcal{R} / I)}(\Gamma(\mathcal{R} / I))$, $\Gamma_{I}(\mathcal{R})$, or $\Gamma(\mathcal{R} / I)$ with some examples clarification. Moreover, we find the degree of each vertex of $T_{I}(\Gamma(\mathcal{R}))$ that depends on the three sets $I, X$, and $Y$. Furthermore, we determine the case when $T_{I}(\Gamma(\mathcal{R}))$ is a complete graph or a regular graph.

Definition 1. Let $\mathcal{R}$ be a commutative ring with $1 \neq 0$ and ideal $I$. Then, a simple graph that is not directed is defined as $T_{I}(\Gamma(\mathcal{R})$ ), possessing vertices of $\mathcal{R}$. In this case, the graph has vertices $x$ and $y$ that are both distinct and adjacent if and only if $x y \in I$.

Proposition 1. (a) If $I=(0)$, then $T_{I}(\Gamma(\mathcal{R}))=\Gamma_{0}(\mathcal{R})$.

(b) If $Z(\mathcal{R})$ is an ideal and $I=Z(\mathcal{R})$, then $T_{I}(\Gamma(\mathcal{R}))=T_{Z(\mathcal{R})}(\Gamma(\mathcal{R}))$.

(c) If $I=\mathcal{R}$, then $T_{I}(\Gamma(\mathcal{R}))=K_{n}$, where $n=|\mathcal{R}|$.

(d) Let $\mathcal{R}$ have a proper nonzero ideal I. Consequently, this means that $T_{Z(\mathcal{R} / I)}(\Gamma(\mathcal{R} / I))=K_{1, n}$. The value of $n$ is given by $n=|\mathcal{R} / I|-1$ if and only if the prime ideal of $\mathcal{R}$ is $I$.

Proof. The proofs of $(a)$ and $(b)$ follow by the definition of the zero-divisor graph of $\mathcal{R}$, which appeared in Beck [4], and definition of the dot total graph of $\mathcal{R}$, which appeared recently in Ashraf et al. [1], respectively.

(c) Let $x$ and $y$ be distinct vertices of $\mathcal{R}$. Then, $x y \in \mathcal{R}=I$. Thus, $x$ is adjacent to $y$ for all $x, y \in \mathcal{R}$. Hence, $T_{I}(\Gamma(\mathcal{R}))=K_{n}$, where $n=|I|=|\mathcal{R}|$. This completes the proof.

(d) Let $I$ be a prime ideal of $\mathcal{R}$. Then, $\mathcal{R} / I$ is an integral domain. Thus, $T_{Z(\mathcal{R} / I)}(\Gamma(\mathcal{R} / I))$ is a star graph. Hence, $T_{Z(\mathcal{R} / I)}(\Gamma(\mathcal{R} / I))=K_{1, n}$, where $n=|\mathcal{R} / I|-1$.

Conversely, let $T_{Z(\mathcal{R} / I)}(\Gamma(\mathcal{R} / I))=K_{1, n}$, where $n=|\mathcal{R} / I|-1$. Then, $T_{Z(\mathcal{R} / I)}(\Gamma(\mathcal{R} / I))$ is a star graph, and we have the following two cases:

Case $(i)$ If $Z(\mathcal{R} / I)=1$, then $\mathcal{R} / I$ is an integral domain.

Case $($ ii $)$ If $Z(\mathcal{R} / I)>1$, then there exists at least two vertices in $Z(\mathcal{R} / I)$. Therefore, $T_{Z(\mathcal{R} / I)}(\Gamma(\mathcal{R} / I))$ is not a star graph, which is a contradiction. 
Thus, we obtain $\mathcal{R} / I$ as an integral domain. Hence, $I$ is the prime ideal of $\mathcal{R}$. This completes the proof.

In view of the following examples, we shall find $T_{I}(\Gamma(\mathcal{R}))$ and $T_{Z(\mathcal{R} / I)}(\Gamma(\mathcal{R} / I))$ with several ideals $I$ of the same ring $\mathcal{R}$.

Example 1. Let $\mathcal{R}=\mathbb{Z}_{8}$. Then, (0),(2),(4), and $\mathbb{Z}_{8}$ are ideals of $\mathcal{R}$ :

(i) Let $I=(0)$, then $\mathcal{R} / I=\mathbb{Z}_{8}$ (see Figure 1 ).
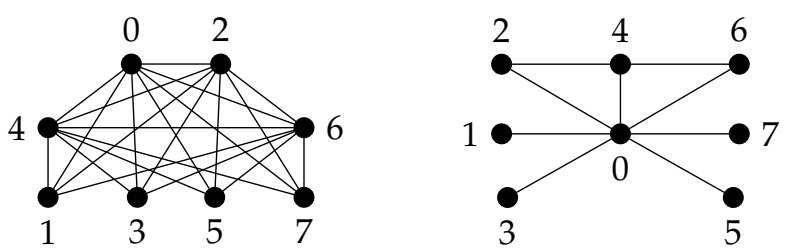

Figure 1. (left) $T_{Z(\mathcal{R} / I)}(\Gamma(\mathcal{R} / I))$ and (right) $T_{I}(\Gamma(\mathcal{R}))$, when $\mathcal{R}=\mathbb{Z}_{8}$ and $I=(0)$.

(ii) Let $I=(2)$, then $\mathcal{R} / I=\mathbb{Z}_{2}$ (see Figure 2 ).
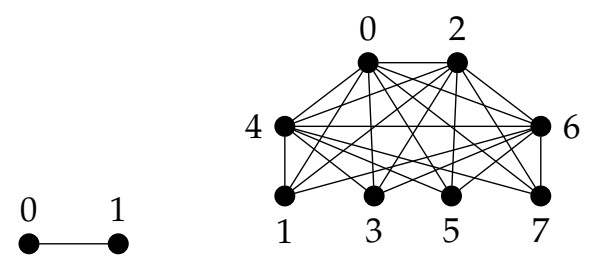

Figure 2. (left) $T_{Z(\mathcal{R} / I)}(\Gamma(\mathcal{R} / I))$ and (right) $T_{I}(\Gamma(\mathcal{R}))$, when $\mathcal{R}=\mathbb{Z}_{8}$ and $I=(2)$.

(iii) $\quad$ Let $I=(4)$, then $\mathcal{R} / I=\mathbb{Z}_{4}$ (see Figure 3 ).
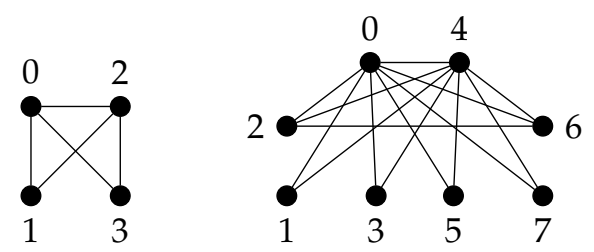

Figure 3. (left) $T_{Z(\mathcal{R} / I)}(\Gamma(\mathcal{R} / I))$ and (right) $T_{I}(\Gamma(\mathcal{R}))$, when $\mathcal{R}=\mathbb{Z}_{8}$ and $I=(4)$.

(iv) Let $I=\mathbb{Z}_{8}$, then $\mathcal{R} / I=(0)$ (see Figure 4 ).

0

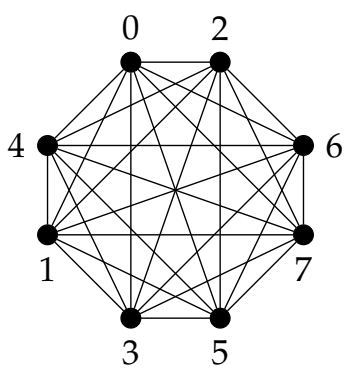

Figure 4. (left) $T_{Z(\mathcal{R} / I)}(\Gamma(\mathcal{R} / I))$ and (right) $T_{I}(\Gamma(\mathcal{R}))$, when $\mathcal{R}=\mathbb{Z}_{8}$ and $I=\mathbb{Z}_{8}$.

Example 2. Let $\mathcal{R}=\mathbb{Z}_{6}$. Then (0), (2), (3), and $\mathbb{Z}_{6}$ are ideals of $\mathcal{R}$ :

(i) Let $I=(0)$, then $\mathcal{R} / I=\mathbb{Z}_{6}$ (see Figure 5). 

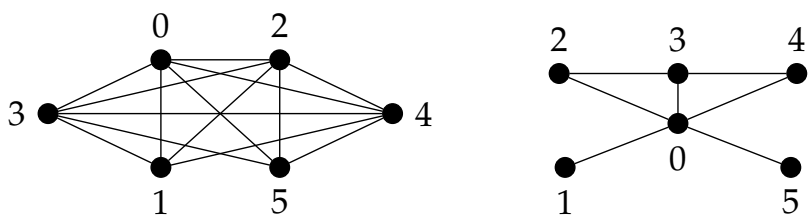

Figure 5. (left) $T_{Z(\mathcal{R} / I)}(\Gamma(\mathcal{R} / I))$ and (right) $T_{I}(\Gamma(\mathcal{R}))$, when $\mathcal{R}=\mathbb{Z}_{6}$ and $I=(0)$.

(ii) $\quad$ Let $I=(2)$, then $\mathcal{R} / I=\mathbb{Z}_{2}$ (see Figure 6).

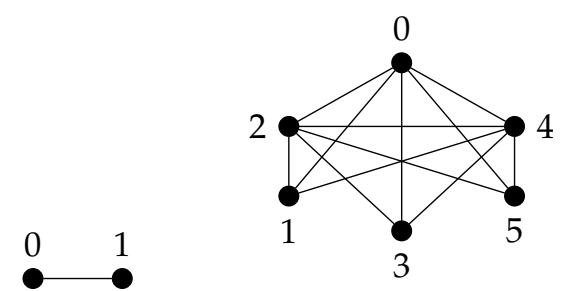

Figure 6. (left) $T_{Z(\mathcal{R} / I)}(\Gamma(\mathcal{R} / I))$ and (right) $T_{I}(\Gamma(\mathcal{R}))$, when $\mathcal{R}=\mathbb{Z}_{6}$ and $I=(2)$.

(iii) $\quad$ Let $I=(3)$, then $\mathcal{R} / I=\mathbb{Z}_{3}$ (see Figure 7 ).
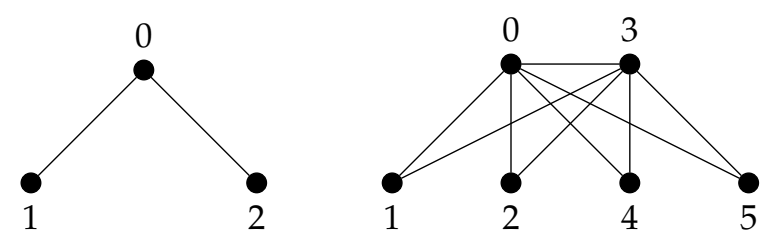

Figure 7. (left) $T_{Z(\mathcal{R} / I)}(\Gamma(\mathcal{R} / I))$ and (right) $T_{I}(\Gamma(\mathcal{R}))$, when $\mathcal{R}=\mathbb{Z}_{6}$ and $I=(3)$.

(iv) $\quad$ Let $I=\mathbb{Z}_{6}$, then $\mathcal{R} / I=(0)$ (see Figure 8).

0

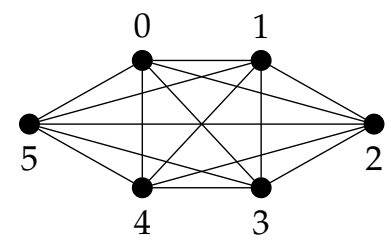

Figure 8. (left) $T_{Z(\mathcal{R} / I)}(\Gamma(\mathcal{R} / I))$ and (right) $T_{I}(\Gamma(\mathcal{R}))$, when $\mathcal{R}=\mathbb{Z}_{6}$ and $I=\mathbb{Z}_{6}$.

Theorem 1. $T_{I}(\Gamma(\mathcal{R}))$ is connected and $\operatorname{diam}\left(T_{I}(\Gamma(\mathcal{R}))\right) \leq 2$. Therefore, where $T_{I}(\Gamma(\mathcal{R}))$ has a cycle, it implies $\operatorname{gr}\left(T_{I}(\Gamma(\mathcal{R}))\right) \leq 5$.

Proof. Assume that $x$ and $y$ are distinct vertices of $T_{I}(\Gamma(\mathcal{R}))$. In such a scenario, various cases will be true as shown:

Case $(i):$ If $x \in I$ and $y \in I$, then $x y \in I$. Thus, $x-y$ is a path of length one in $T_{I}(\Gamma(\mathcal{R}))$.

Case(ii): If $x \in I$ and $y \notin I$, then $x y \in I$. Thus, $x-y$ is a path of length one in $T_{I}(\Gamma(\mathcal{R}))$.

Case(iii): If $x \notin I$ and $y \in I$, then this will result in a path of length one as in the previous case.

Case (iv): If $x \notin I$ and $y \notin I$, then we will consider the following subcases:

Subcase $(a)$ : If $x y \in I$, then $x-y$ is a path of length one in $T_{I}(\Gamma(\mathcal{R}))$.

Subcase $(b)$ : If $x y \notin I$, then there is some $z \in I$ such that $x z \in I$ and $z y \in I$. Thus, $x-z-y$ is a path of length two in $T_{I}(\Gamma(\mathcal{R}))$. Hence, $T_{I}(\Gamma(\mathcal{R}))$ is connected and $\operatorname{diam}\left(T_{I}(\Gamma(\mathcal{R}))\right) \leq 2$. Since for any undirected graph, $H$ contains a cycle, $g r(H) \leq 2$ $\operatorname{diam}(H)+1$ (for reference see [8]). Thus, $g r\left(T_{I}(\Gamma(\mathcal{R}))\right) \leq 5$. 
Suppose that $\mathcal{R}$ is a commutative ring, and $I$ is an ideal of $\mathcal{R}$. We construct a graph $T_{I}(\Gamma(\mathcal{R}))$ with the following method:

First, the set of vertices of $T_{I}(\Gamma(\mathcal{R}))$ can be classified into three disjoint subsets of $\mathcal{R}$ :

(i) $I=(a)$ is the subset of $\mathcal{R}$ such that $I$ is the ideal generated by the element $a$.

(ii) $X=\{x \in \mathcal{R} \backslash I: x b \notin I$, for all $b \in \mathcal{R} \backslash I\}$.

(iii) $Y=\{y \in \mathcal{R} \backslash I: y b \in I$, for some $b \in \mathcal{R} \backslash I\}$.

Second, we will connect the edges between the vertices defined in the three previous sets as follows:

We define a complete graph $\left(K_{n}\right.$, where $\left.n=|I|\right)$ by using the first set $I=(a)$ as its vertex set. Thus, we have an edge between each vertex of $I$ (i.e., $a b \in I$ ). Then, join each vertex of the second set $X$ to all vertices of the complete graph $K_{n}$, and similarly, join each vertex of the third set $Y$ to all vertices of the complete graph $K_{n}$. Thus, we have an edge between each vertex of the sets $X$ and $Y$ with all vertex of $I$ (i.e., $x a \in I$ and $y a \in I$ ). Finally, in this part of the edges the relationship between $T_{I}(\Gamma(\mathcal{R}))$ and $\Gamma_{I}(\mathcal{R})$ is identical. Thus, for distinct $y_{1}, y_{2} \in Y, y_{1}$ is adjacent to $y_{2}$ in $T_{I}(\Gamma(\mathcal{R}))$ if and only if $y_{1}$ is adjacent to $y_{2}$ in $\Gamma_{I}(\mathcal{R})$ (i.e., $y_{1} y_{2} \in I$ ).

Henceforth, we shall rely on the three sets $I, X$, and $Y$ defined above in this paper.

Theorem 2. Let $y_{1}$ and $y_{2}$ be any two distinct vertices of the third set $Y$ and $y_{1}$ is adjacent to $y_{2}$ in $T_{I}(\Gamma(\mathcal{R}))$. If $y_{1}+I \neq y_{2}+I$, then $y_{1}+I$ is adjacent to $y_{2}+I$ in $\Gamma(\mathcal{R} / I)$ and if $y_{1}+I=y_{2}+I$, then $y_{1}^{2}, y_{2}^{2} \in I$.

Corollary 1. Let $T_{I}(\Gamma(\mathcal{R})$ ) have two vertices ( $u$ and $v$ ) that are both distinct and adjacent. This implies that the elements, $u+I$ and $v+I$, are adjacent in $T_{I}(\Gamma(\mathcal{R}))$. Assuming that $u^{2} \in I$, this implies that $T_{I}(\Gamma(\mathcal{R}))$ has all the distinct elements of $u+I$ adjacent to it.

Corollary 2. Let I be an ideal of $\mathcal{R}$. Then, $\Gamma(\mathcal{R} / I)$ and $\Gamma_{I}(\mathcal{R})$ are subgraphs of $T_{I}(\Gamma(\mathcal{R}))$.

Corollary 3. Assume that a nonzero ideal of $\mathcal{R}$ is $I$. Then, if and only if the prime ideal of $\mathcal{R}$ is $I$, $T_{Z(\mathcal{R} / I)}(\Gamma(\mathcal{R} / I))$ is subgraph of $T_{I}(\Gamma(\mathcal{R}))$.

Corollary 4. $T_{I}(\Gamma(\mathcal{R}))$ contains $|I|$ disjoint subgraphs isomorphic to $\Gamma(\mathcal{R} / I)$.

Proof. Since $\Gamma_{I}(\mathcal{R})$ is subgraph of $T_{I}(\Gamma(\mathcal{R}))$ (see Corollary 2) and $\Gamma_{I}(\mathcal{R})$ contains $|I|$ disjoint subgraphs isomorphic to $\Gamma(\mathcal{R} / I), T_{I}(\Gamma(\mathcal{R}))$ contains $|I|$ disjoint subgraphs isomorphic to $\Gamma(\mathcal{R} / I)$.

Remark 1. Let $I, X$, and $Y$ be three disjoint sets defined above, and $u, v \in \mathcal{R}$. Then, we have the following results:

(i) If $u+I$ is adjacent to $v+I$ in $\Gamma(\mathcal{R} / I)$, then $u$ is adjacent to $v$ in $T_{I}(\Gamma(\mathcal{R})$ ).

(ii) If $u+I$ is adjacent to $v+I$ in $T_{Z(\mathcal{R} / I)}(\Gamma(\mathcal{R} / I))$, then $u$ may or may not be adjacent to $v$ in $T_{I}(\Gamma(\mathcal{R})$ ) (see Example 1(iii); Figure 3).

(iii) If I is a prime ideal, then the set $Y$ defined above will vanish.

(iv) If $I=\mathcal{R}$, then the sets $X$ and $Y$ defined above will vanish, i.e., $X$ and $Y$ are empty. Thus, $T_{I}(\Gamma(\mathcal{R}))=K_{n}$, where $n=|\mathcal{R}|$.

(v) If $u \in I$, then $u$ is adjacent to each vertex $v \in \mathcal{R}$.

(vi) If $u \in X$, then $u$ is adjacent to $v \in$ I only.

(vii) If $u \in Y$, then $u$ is adjacent to $v \in I$ and some $v \in Y$.

(viii) Any two distinct vertices of $X$ are not adjacent in $T_{I}(\Gamma(\mathcal{R}))$, i.e., if $u, v \in X$, and $u \neq v$, then $u v \notin E\left(T_{I}(\Gamma(\mathcal{R}))\right)$.

(ix) If $Y$ is a subgraph of $T_{I}(\Gamma(\mathcal{R}))$, then each pair of distinct vertices $u$ and $v$ of $Y$ is connected by a path with a length of at most three.

$(x) \quad$ There are no adjacencies between elements of $X$ and elements of $Y$. 
Theorem 3. Let I be a prime ideal of $\mathcal{R}$. Then, $T_{I}(\Gamma(\mathcal{R}))$ contains a subgraph isomorphic to $K_{n}$, where $n=|I|+1$.

Proof. Since $I$ is a prime ideal, $Y$ will vanish, and there is at least one element $v$ in $X$. Moreover, we have a complete subgraph $K_{n}$, where $n=|I|$ and the vertex $v$ is adjacent to each vertex of $K_{n}$. Thus, we have a complete subgraph of order $n=|I|+1$.

Theorem 4. Let I be a non-prime ideal of $\mathcal{R}$, and there exists $u \in Y$ such that $u^{2} \in I$. Then, $T_{I}(\Gamma(\mathcal{R}))$ contains a subgraph isomorphic to $K_{m}$, where $m$ is at least $2|I|$.

Proof. Since there exists $u \in Y$ such that $u^{2} \in I$, all the distinct elements of $u+I$ are adjacent in $T_{I}(\Gamma(\mathcal{R})$ ) (see Corollary 1 ). Thus, we have a complete subgraph of order $n=|I|$. Moreover, by element of the set $I$, we have a complete subgraph $K_{n}$, where $n=|I|$ and all the distinct elements of $u+I$ are adjacent to each vertex of $K_{n}$. Thus, we have a complete subgraph of order $m=n+n=|I|+|I|=2|I|$. Therefore, if $Y$ consists of the elements $u+I$ only, then $m=2|I|$. If there exists $v \in Y$ other than the elements $u+I$, then $v$ is adjacent to all the elements $u+I$ or there exists an element $w \in Y$ such that $v$ is adjacent to $w$, and $w$ is adjacent to all the elements $u+I$. Hence, in both cases we have a complete subgraph of order $|I|+1$, and all elements of this subgraph are adjacent to each vertex of $K_{n}$. Thus, we have a complete subgraph of order $m=|I|+1+|I|=2|I|+1$.

Theorem 5. Let I be an ideal of $\mathcal{R}$ that is not prime, and $u^{2} \notin I$ for all $u \in Y$. Then, $T_{I}(\Gamma(\mathcal{R}))$ contains a subgraph isomorphic to $K_{m}$, where $m$ is at least $|I|+1$.

Proof. Assume that $u^{2} \notin I$ for all $u \in Y$. By the same arguments as used in Theorems 3 and 4 , we obtain the result.

Corollary 5. $T_{I}(\Gamma(\mathcal{R}))$ is a complete graph if and only if either $I=\mathcal{R}$ or $\mathcal{R} \cong \mathbb{Z}_{2}$.

Theorem 6. Let I be a prime ideal of $\mathcal{R}$. Then, the degree of each vertex of $T_{I}(\Gamma(\mathcal{R}))$ is either $|\mathcal{R}|-1$ or $|I|$.

Proof. Since $I$ is a prime ideal of $\mathcal{R}$, the set $Y$ will vanish. Thus, $\mathcal{R}$ consists of two disjoint subsets $I$ and $X$. Then, we have the following two cases:

Case $(i):$ If $u \in I$, then $u$ is adjacent to each vertex in $T_{I}(\Gamma(\mathcal{R}))$ except $u$; that is, $u$ is adjacent to $(|\mathcal{R}|-1)$ vertices, and hence the degree of $u$ is $|\mathcal{R}|-1$.

Case (ii): If $u \in X$, then $u$ is adjacent to the vertices, which belongs to $I$; that is, $u$ is adjacent to $|I|$ vertices and, hence, $\operatorname{deg}(u)=|I|$.

Corollary 6. Let I be an ideal of $\mathcal{R}$ and $u \in Y$. Then, the number of elements of $T_{I}(\Gamma(\mathcal{R}))$ adjacent to $u$ is either $|I|$ or at least $|I|+1$.

Proof. Let $u \in Y$. Then, we have two types of adjacencies. First, $u$ is adjacent to each element of $I$, i.e., $u$ is adjacent to $|I|$ vertices. Second, if $|Y| \geq 2$, then $u$ is adjacent to some elements of $Y$, i.e., $u$ is adjacent to at least one vertex of $Y$. Thus, $u$ is adjacent to at least $|I|+1$ vertices. If $|Y|=1$, then $u$ is adjacent to $|I|$ elements only. Hence, $\operatorname{deg}(u) \geq|I|+1$ or $\operatorname{deg}(u)=|I|$.

Corollary 7. Let I be an ideal of $\mathcal{R}$ and $u \in T_{I}(\Gamma(\mathcal{R}))$. Then, the degree of $u$ depends on the three sets $I, X$, and $Y$ defined earlier as follows.

$$
\operatorname{deg}(u)= \begin{cases}|\mathcal{R}|-1 & \text { if } u \in I \\ |I| & \text { if } u \in X \text { or } u \in Y \text { and }|Y|=1 \\ \text { at least }|I|+1 & \text { if } u \in Y \text { and }|Y| \geq 2\end{cases}
$$


Corollary 8. $T_{I}(\Gamma(\mathcal{R}))$ is regular graph if and only if either $I=\mathcal{R}$ or $\mathcal{R} \cong \mathbb{Z}_{2}$ (i.e., $T_{I}(\Gamma(\mathcal{R})$ ) is a complete graph).

Remark 2. Minimum degree of $T_{I}(\Gamma(\mathcal{R}))$ is $\delta\left(T_{I}(\Gamma(\mathcal{R}))\right)=|I|$, and maximum degree of $T_{I}(\Gamma(\mathcal{R}))$ is $\Delta\left(T_{I}(\Gamma(\mathcal{R}))\right)=|\mathcal{R}|-1$.

\section{Connectivity of $T_{I}(\Gamma(\mathcal{R}))$}

In this section, we study the connectivity of $T_{I}(\Gamma(\mathcal{R}))$.

Theorem 7. Let I be a nonzero ideal of $\mathcal{R}$. Then, $T_{I}(\Gamma(\mathcal{R}))$ has no cut-vertex.

Proof. Assume that the vertex $u$ of $T_{I}(\Gamma(\mathcal{R}))$ is a cut-vertex. Then, there exist $x, y \in$ $T_{I}(\Gamma(\mathcal{R}))$ such that $u$ lies on every path from $x$ to $y$. Thus, we have the following cases.

Case (i): If $x$ is adjacent to $y$, then there is a path from $x$ to $y$ in $T_{I}(\Gamma(\mathcal{R}))$ such that $u$ does not lie on it. Hence, we obtain a contradiction.

Case (ii): If $x$ is not adjacent to $y$, then $x, y \notin I$. Since $I$ is nonzero ideal, $I$ has at least two elements, and we have the following subcases.

Subcase $(a):$ If $x, y \in X$, then $x$ is not adjacent to $y$, and there exist $w_{1}, w_{2} \in I$ such that $x$ is adjacent to $w_{1}, w_{2}$, and similarly, $y$ is adjacent to $w_{1}, w_{2}$. Therefore, if $u$ is equal to $w_{1}$ or $w_{2}$, then there is at least one path from $x$ to $y$, and $u$ does not lie on it, which is a contradiction. Moreover, we obtain the same contradiction when $u$ is not equal to $w_{1}$ and $w_{2}$.

Subcase (b): If $x \in X$ and $y \in Y$, then $x$ is not adjacent to $y$, and by the same arguments as used in the above subcase, we obtain the contradiction.

Subcase (c): If $x, y \in Y$, then $x$ may or may not adjacent to $y$. Thus in both the cases and by the same arguments as used in the subcase $(a)$, we obtain a contradiction.

Corollary 9. Let I be an ideal of $\mathcal{R}$. Then, $T_{I}(\Gamma(\mathcal{R}))$ has a cut-vertex if and only if I is a zero ideal.

Remark 3. If $T_{I}(\Gamma(\mathcal{R}))$ has a cut-vertex, then 0 is the cut-vertex of $T_{I}(\Gamma(\mathcal{R}))$.

Theorem 8. $k\left(T_{I}(\Gamma(\mathcal{R}))\right)=|I|$.

Proof. By Remark $2, \delta\left(T_{I}(\Gamma(\mathcal{R}))\right)=|I|$. Moreover, for any graph $G, k(G) \leqslant \lambda(G) \leqslant \delta(G)$. Therefore, $k\left(T_{I}(\Gamma(\mathcal{R}))\right) \leqslant|I|$. Now, if $u \in I$, then $u$ is adjacent to each vertex $v \in T_{I}(\Gamma(\mathcal{R}))$. Thus, the minimum vertex-cut is the set of all those vertices in $I$. Therefore, $k\left(T_{I}(\Gamma(\mathcal{R}))\right) \geq$ $|I|$, and hence $k\left(T_{I}(\Gamma(\mathcal{R}))\right)=|I|$.

Remark 4. For any commutative ring $\mathcal{R}$ with $1 \neq 0$, the elements of I with some elements of $Y$ form a vertex-cut of $T_{I}(\Gamma(\mathcal{R}))$. However, only the elements of $I$ is the minimum vertex-cut of $T_{I}(\Gamma(\mathcal{R}))$.

Theorem 9. $T_{I}(\Gamma(\mathcal{R}))$ has a bridge if and only if either $T_{I}(\Gamma(\mathcal{R}))$ is a graph with two vertices (i.e., $T_{I}(\Gamma(\mathcal{R})) \cong T_{I}\left(\Gamma\left(\mathbb{Z}_{2}\right)\right)$ ), or I is the zero ideal of $\mathcal{R}$.

Proof. Suppose that $T_{I}(\Gamma(\mathcal{R}))$ has a bridge. Now, we have the following cases.

Case $(i)$ : If $|\mathcal{R}|=2$, then it is clear that $T_{I}(\Gamma(\mathcal{R})) \cong K_{2}$, which has a bridge. Hence, $T_{I}(\Gamma(\mathcal{R})) \cong T_{I}\left(\Gamma\left(\mathbb{Z}_{2}\right)\right)$.

Case $(i i)$ : If $|\mathcal{R}| \geqslant 3$, then either $V\left(T_{I}(\Gamma(\mathcal{R}))\right) \subseteq I, V\left(T_{I}(\Gamma(\mathcal{R}))\right) \subseteq X$, or $V\left(T_{I}(\Gamma(\mathcal{R}))\right) \subseteq$ $Y$. Let $e=u v$ be the bridge of $T_{I}(\Gamma(\mathcal{R}))$. Since there is no edge neither between any two elements of $X$ nor between any element of $X$ with element of $Y$, we have the following subcases.

Subcase $(a)$ : If $u, v \in I$, and $|\mathcal{R}| \geqslant 3$, then there exists $w \in \mathcal{R}$ such that $u$ and $v$ are adjacent to $w$. We note that $u-v-w-u$ is a cycle, and there is no bridge between them; we obtain a contradiction. 
Subcase $(b):$ If $u, v \in Y$, and $|\mathcal{R}| \geqslant 3$, then there exists $w \in I$ such that $u$ and $v$ are adjacent to $w$. We note that $u-v-w-u$ is a cycle, and there is no bridge between them; we obtain a contradiction.

Subcase (c): If $u \in I, v \in X$, and $|\mathcal{R}| \geqslant 3$, then there are two possibilities.

If $|I|=1$ (i.e., $I$ is a zero ideal of $\mathcal{R}$ ), then $v$ is adjacent to $u=0$ only, and $u v$ is a bridge of $T_{I}(\Gamma(\mathcal{R}))$. Moreover, each vertex of $X$ with $u$ forms a bridge of $T_{I}(\Gamma(\mathcal{R})$ ) (i.e., we have $|X|$ bridges $)$.

If $|I| \geq 2$ (i.e., $I$ is not zero ideal of $\mathcal{R}$ ), then there exists $u \neq w \in I$ such that $u$ and $v$ are adjacent to $w$. We note that $u-v-w-u$ is a cycle, and there is no bridge between them; we obtain a contradiction.

Subcase $(d)$ : If $u \in I, v \in Y$, and $|\mathcal{R}| \geqslant 3$, then there are three possibilities.

If $|I|=1$ (i.e., $I$ is a zero ideal of $\mathcal{R}$ ) and $|Y|=1$, then $v$ is adjacent to $u=0$ only, and $u v$ is a bridge of $T_{I}(\Gamma(\mathcal{R}))$.

If $|I|=1$ (i.e., $I$ is a zero ideal of $\mathcal{R}$ ) and $|Y| \geq 2$, then there exists at least one element $v \neq w \in Y$ such that $v$ and $w$ are connected vertices by a path $P$. Since each elements of $Y$ are adjacent to elements of $I, v-P-w-0-v$ is a cycle and there is no bridge between them. This is a contradiction.

If $|I| \geq 2$ (i.e., $I$ is not zero ideal of $\mathcal{R}$ ), then there exists $u \neq w \in I$ such that $v$ is adjacent to $w$. Since each element of $I$ is adjacent, $u-v-w-u$ is a cycle, and there is no bridge between them; we obtain a contradiction.

Conversely, suppose that $T_{I}(\Gamma(\mathcal{R}))$ is a graph with two vertices. Then, it is clear that $T_{I}(\Gamma(\mathcal{R}))$ has a bridge. Let us suppose that $I$ is the zero ideal of $\mathcal{R}$ (i.e., $I=\{0\}$ ) and $|\mathcal{R}| \geq 3$. Then, we have at least one element $u$ in $X$. Hence, $0 u$ is a bridge in $T_{I}(\Gamma(\mathcal{R}))$.

Remark 5. If the ring $\mathcal{R} \cong \mathbb{Z}_{2}$ or $I$ is the zero ideal of $\mathcal{R}$, then $T_{I}(\Gamma(\mathcal{R}))$ has a bridge and vice versa, i.e., if $T_{I}(\Gamma(\mathcal{R}))$ has a bridge, then the ring $\mathcal{R} \cong \mathbb{Z}_{2}$ or $I$ is the zero ideal of $\mathcal{R}$.

\section{Clique Number and Girth of $T_{I}(\Gamma(\mathcal{R}))$}

In this section, we study the clique number and girth of $T_{I}(\Gamma(\mathcal{R}))$.

Theorem 10. Let $I$ be a prime ideal of $\mathcal{R}$ and $|I|=m$. Then, $T_{I}(\Gamma(\mathcal{R}))$ has cliques of the form $K_{1}, K_{2}, K_{3}, \ldots, K_{m+1}$. Moreover, $\omega\left(T_{I}(\Gamma(\mathcal{R}))\right)=m+1$.

Proof. Suppose that $I$ is a prime ideal of $\mathcal{R}$ and $|I|=m$. Then, by using Theorem 3, $T_{I}(\Gamma(\mathcal{R}))$ contains a complete subgraph of order $|I|+1$, and this order is the greatest integer $n=|I|+1 \geq 2$ such that $K_{n} \subseteq T_{I}(\Gamma(\mathcal{R}))$. Hence, $\omega\left(T_{I}(\Gamma(\mathcal{R}))\right)=|I|+1=m+1$.

Theorem 11. Let $I$ be an ideal of $\mathcal{R}$, which is not prime and there exists $u \in Y$ such that $u^{2} \in I$. Then, $\omega\left(T_{I}(\Gamma(\mathcal{R}))\right) \geq 2|I|$.

Proof. Suppose that $I$ is not a prime ideal of $\mathcal{R}$. Then, by using Theorem $4, T_{I}(\Gamma(\mathcal{R}))$ contains a complete subgraph of order at least $2|I|$. Hence, $\omega\left(T_{I}(\Gamma(\mathcal{R}))\right) \geq 2|I|$.

Theorem 12. Let $I$ be an ideal of $\mathcal{R}$, which is not prime, and $u^{2} \notin I$ for all $u \in Y$. Then, $\omega\left(T_{I}(\Gamma(\mathcal{R}))\right) \geq|I|+1$.

Proof. Suppose that $I$ is not a prime ideal of $\mathcal{R}$. Then, by using Theorem $5, T_{I}(\Gamma(\mathcal{R}))$ contains a complete subgraph of order at least $|I|+1$. Hence, $\omega\left(T_{I}(\Gamma(\mathcal{R}))\right) \geq|I|+1$.

Corollary 10. Let I be a non-prime ideal of $\mathcal{R}$ and $|I|=n$. Then, $\omega\left(T_{I}(\Gamma(\mathcal{R}))\right)=\omega\left(\Gamma_{I}(\mathcal{R})\right)+n$.

Remark 6. Let $I$ be an ideal of a ring $\mathcal{R}$. Then, $\omega(\Gamma(\mathcal{R} / I)) \leq \omega\left(\Gamma_{I}(\mathcal{R})\right) \leq \omega\left(T_{I}(\Gamma(\mathcal{R}))\right)$. Moreover, we know that if $\Gamma_{I}(\mathcal{R})$ has no connected columns (i.e., if $u^{2} \notin I$ for all $u \in Y$ ), then $\omega(\Gamma(\mathcal{R} / I))=\omega\left(\Gamma_{I}(\mathcal{R})\right)$ (for reference see Theorem $\left.4.5[2]\right)$. 
Theorem 13. Let I be a nonzero ideal of a ring $\mathcal{R}$. If $|\mathcal{R}| \geq 3$, then $T_{I}(\Gamma(\mathcal{R}))$ has a cycle. Moreover, $\operatorname{gr}\left(T_{I}(\Gamma(\mathcal{R}))\right)=3$.

Proof. Since $I$ is a nonzero ideal, $I$ has at least two elements (say $u, v$ ). Moreover, each element of $\mathcal{R}$ is adjacent to the elements of $I$ and $|\mathcal{R}| \geq 3$, i.e., there exists $w \in \mathcal{R}$ such that $u$ and $v$ are adjacent to $w$. Thus, $u-w-v-u$ is a cycle of length three, which is the smallest cycle in $T_{I}(\Gamma(\mathcal{R}))$. Hence, $g r\left(T_{I}(\Gamma(\mathcal{R}))\right)=3$.

Corollary 11. Let I be an ideal of a ring $\mathcal{R}$. If $|\mathcal{R}| \leq 2$, then $\operatorname{gr}\left(T_{I}(\Gamma(\mathcal{R}))\right)=\infty$.

Corollary 12. Let I be a zero ideal of a ring $\mathcal{R}$. Then,

$$
\operatorname{gr}\left(T_{I}(\Gamma(\mathcal{R}))\right)= \begin{cases}3 & \text { if }|Y| \geq 2 \\ \infty & \text { if }|Y| \leq 1\end{cases}
$$

Remark 7. Let I be a non-prime ideal of a ring $\mathcal{R}$. Then,

$$
g r\left(T_{I}(\Gamma(\mathcal{R}))\right) \leq g r\left(\Gamma_{I}(\mathcal{R})\right) \leq g r(\Gamma(\mathcal{R} / I)) .
$$

\section{5. $T_{I}(\Gamma(\mathcal{R}))$ Is Eulerian and Hamiltonian}

In this section, we determine when $T_{I}(\Gamma(\mathcal{R}))$ is Eulerian, Hamiltonian, and $T_{I}(\Gamma(\mathcal{R}))$ contains a Eulerian trail.

Theorem 14. Let $I$ be an ideal of a ring $\mathcal{R}$ such that $|\mathcal{R}|=n \geq 3$. If $I=\mathcal{R}$ and $|\mathcal{R}|$ are odd, then $T_{I}(\Gamma(\mathcal{R}))$ is a Eulerian.

Proof. Suppose that $I=\mathcal{R}$ and $|\mathcal{R}|$ is odd. Then, by Corollary $5, T_{I}(\Gamma(\mathcal{R}))$ is a complete graph $K_{n}$ of odd vertices. Thus, the degree of each vertex of $T_{I}(\Gamma(\mathcal{R}))$ is even. Hence, $T_{I}(\Gamma(\mathcal{R}))$ is Eulerian.

Theorem 15. Let I be a zero ideal of a ring $\mathcal{R}$ such that $|X|=1$ and $|Y|$ are even. If each vertex of $\Gamma(\mathcal{R})=\Gamma_{0}(\mathcal{R})$ has odd degree, then $T_{I}(\Gamma(\mathcal{R}))$ contains a Eulerian trail.

Proof. Suppose that each vertex of $\Gamma(\mathcal{R})=\Gamma_{0}(\mathcal{R})$ has odd degree. Then, each vertex of $Y$ in $T_{I}(\Gamma(\mathcal{R}))$ has even degree. Since $|X|=1$ and $|Y|$ are even, the vertex 0 of $I$ has odd degree. Moreover, the degree of the vertex of $X$ has degree one. Thus, each vertex of $T_{I}(\Gamma(\mathcal{R}))$ has an even degree except for two vertices that have odd degrees. Hence, $T_{I}(\Gamma(\mathcal{R}))$ contains a Eulerian trail.

Remark 8. In view of Theorem 15, the element of $X$ is the unity of the ring $\mathcal{R}$. Moreover, the Eulerian trail of $T_{I}(\Gamma(\mathcal{R}))$ begins at unity and ends at zero of $\mathcal{R}$ or begins at zero and ends at unity of $\mathcal{R}$ (for example, $\mathcal{R}=\mathbb{Z}_{2} \times \mathbb{Z}_{2}$ and $I=(0)$ ).

Theorem 16. Assume that a prime ideal of Ring $\mathcal{R}$ is $I$. Thus, if and only if $|I|$ is even and $|X|$ is odd, then $T_{I}(\Gamma(\mathcal{R}))$ is Eulerian. $|\mathcal{R}|$ also is odd.

Proof. Suppose that $T_{I}(\Gamma(\mathcal{R}))$ is Eulerian. Then, every vertex of $T_{I}(\Gamma(\mathcal{R}))$ has an even degree. Since $I$ is a prime ideal of $\mathcal{R}$, the degree of each vertex of $T_{I}(\Gamma(\mathcal{R}))$ either $(|\mathcal{R}|-1)$ or $|I|$ (Theorem 6). Therefore, we have the following cases.

Case $(i)$ : If $u \in X$, then $\operatorname{deg}(u)=|I|$, which is even. Thus, $|I|$ is even.

Case (ii): If $u \in I$, then $\operatorname{deg}(u)=|\mathcal{R}|-1$, which is even, and we obtain $|\mathcal{R}|$ as odd.

Now, we have $|\mathcal{R}|$ as odd and $|I|$ as even. Therefore, $|X|$ is odd.

Conversely, suppose that $|I|$ is even and $|X|$ is odd. Then, $|\mathcal{R}|$ is odd. Thus, $|\mathcal{R}|-1$ is even, and $|I|$ is also even. Since $I$ is a prime ideal of $\mathcal{R}$, the degree of each vertex of 
$T_{I}(\Gamma(\mathcal{R}))$ is either $|\mathcal{R}|-1$ or $|I|$. Thus, the degree of each vertex of $T_{I}(\Gamma(\mathcal{R}))$ is even. Hence, $T_{I}(\Gamma(\mathcal{R}))$ is Eulerian.

Theorem 17. Let I be a prime ideal of a ring $\mathcal{R}$. Then, $T_{I}(\Gamma(\mathcal{R}))$ contains a Eulerian trail if and only if either $|I|=2$ or $|X|$ is even or $|X|=2$ and $|I|$ are odd.

Proof. Suppose that $T_{I}(\Gamma(\mathcal{R}))$ contains a Eulerian trail. Then, exactly two vertices of $T_{I}(\Gamma(\mathcal{R}))$ have odd degree. Since $I$ is a prime ideal of $\mathcal{R}$, the vertex set of $T_{I}(\Gamma(\mathcal{R}))$ consists of $I$ and $X$ only, and $Y$ will vanish. Let $u$ and $v$ be the two vertices of odd degree and let $w_{1}, w_{2}, \ldots, w_{n}$ be the vertices of even degree. Then, we have the following cases.

Case $(i):$ If $u, v \in I$ and $w_{i} \in X$ for all $1 \leq i \leq n$, then $\operatorname{deg}(u)=\operatorname{deg}(v)$ is odd and $\operatorname{deg}\left(w_{i}\right)$ for all $1 \leq i \leq n$ is even. Therefore, $|\mathcal{R}|-1$ is odd, and $|I|=2$ is even; thus, $|\mathcal{R}|$ is even and $|I|=2$. Hence, $|I|=2$ and $|X|$ is even. Moreover, $|\mathcal{R}|$ is even.

Case(ii): If $u, v \in I$ and there exists at least one $w_{j} \in I$, then $\operatorname{deg}(u)=\operatorname{deg}(v)=$ $\operatorname{deg}\left(w_{j}\right)$ is odd. Hence, there are more than two odd vertices in $T_{I}(\Gamma(\mathcal{R}))$, and we obtain a contradiction.

Case(iii): If $u, v \in X$ and $w_{i} \in I$ for all $1 \leq i \leq n$, then $\operatorname{deg}(u)=\operatorname{deg}(v)$ is odd and $\operatorname{deg}\left(w_{i}\right)$ for all $1 \leq i \leq n$ is even. Note that $|I|$ is odd, and $|\mathcal{R}|-1$ is even. We obtain $|I|$ as odd and $|\mathcal{R}|$ as odd. Since $u, v \in X$ only, we have $|X|=2$. Hence, $|X|=2$ and $|I|$ are odd. Moreover, $|\mathcal{R}|$ is odd.

Case (iv): If $u, v \in X$ and there exists at least one $w_{j} \in X$, then $\operatorname{deg}(u)=\operatorname{deg}(v)=$ $\operatorname{deg}\left(w_{j}\right)$ is odd. Thus, there are more than two odd vertices in $T_{I}(\Gamma(\mathcal{R}))$, we obtain a contradiction.

Case $(v)$ : If $u \in I$ and $v \in X$, then $\operatorname{deg}(u)=\operatorname{deg}(v)=\operatorname{deg}\left(w_{i}\right)$ for all $1 \leq i \leq n$ is odd. Thus, all the vertices of $T_{I}(\Gamma(\mathcal{R}))$ have odd degree, and we obtain a contradiction. Therefore in all the cases, we obtain either $|I|=2$ and $|X|$ as even or $|X|=2$ and $|I|$ as odd.

Conversely, suppose that either $|I|=2$ and $|X|$ are even or $|X|=2$ and $|I|$ are odd. We first assume that $|I|=2$ and $|X|$ are even. Thus, $|\mathcal{R}|$ is even, and let $u$ be any vertex of $T_{I}(\Gamma(\mathcal{R}))$. Therefore, we have the following cases.

Case $(i)$ : If $u \in I$, then $\operatorname{deg}(u)=|\mathcal{R}|-1$, which is odd. Since $|I|=2$ and $|X|$ are even, and there are only two vertices in I posessing an odd degree, and each vertices in $X$ has an even degree. Hence, $T_{I}(\Gamma(\mathcal{R}))$ contains a Eulerian trail.

Case(ii): If $u \in X$, then $\operatorname{deg}(u)=|I|=2$, which is even by the same argument; there are only two vertices $w_{1}, w_{2} \in I$ such that $w_{1}$ and $w_{2}$ are adjacent to each vertices in $X$ and $w_{1}$ adjacent to $w_{2}$ and $\operatorname{deg}\left(w_{1}\right)=\operatorname{deg}\left(w_{2}\right)=|X|+1$, which is odd. Therefore, there are only two vertices in $I$ that have an odd degree, and each other vertices in $X$ has even degree. Hence, $T_{I}(\Gamma(\mathcal{R}))$ contains a Eulerian trail.

Now, we assume that $|X|=2$ and $|I|$ are odd. Thus, $|\mathcal{R}|$ is odd, and let $u$ be any vertex of $T_{I}(\Gamma(\mathcal{R}))$. Then, we have the following cases.

Case $(i)$ : If $u \in I$, then $\operatorname{deg}(u)=|\mathcal{R}|-1$, which is even. Since $|X|=2$ and $|I|$ are odd, there are only two vertices in $X$ that have an odd degree, and each other vertices in $I$ has an even degree. Hence, $T_{I}(\Gamma(\mathcal{R}))$ contains a Eulerian trail.

Case(ii): If $u \in X$, then $\operatorname{deg}(u)=|I|$, which is odd; thus, $|\mathcal{R}|$ is odd. By the same argument, there are only two vertices in $X$ possessing an odd degree and each other vertices in $I$ has degree $|\mathcal{R}|-1$, which is even. Hence, $T_{I}(\Gamma(\mathcal{R})$ ) contains a Eulerian trail.

From all the above cases, we conclude that $T_{I}(\Gamma(\mathcal{R}))$ contains a Eulerian trail. Hence, if either $|I|=2$ and $|X|$ are even or $|X|=2$ and $|I|$ are odd, then $T_{I}(\Gamma(\mathcal{R}))$ contains a Eulerian trail.

Remark 9. In view of Theorem 17, if $|I|=2$, then Eulerian trail of $T_{I}(\Gamma(\mathcal{R}))$ begins at one of these two elements of $I$ and ends at other(for example $\mathcal{R}=\mathbb{Z}_{6}$ and $I=(3)$ see Example 2(iii) Figure 7). Moreover, if $|X|=2$, then the Eulerian trail of $T_{I}(\Gamma(\mathcal{R}))$ begins at one of these two elements of $X$ and ends at the other (for example $\mathcal{R}=\mathbb{Z}_{3}$ and $I=(0)$ ). 
Theorem 18. Let I be an ideal of a ring $\mathcal{R}$ that is not prime such that $|I|$ is even, $|X|$ is odd, and $|Y|$ is even. Then, we have the following case:

(a) $T_{I}(\Gamma(\mathcal{R}))$ is Eulerian if and only if $\Gamma_{I}(\mathcal{R})$ is Eulerian.

(b) $T_{I}\left(\Gamma(\mathcal{R})\right.$ ) contains a Eulerian trail if and only if $\Gamma_{I}(\mathcal{R})$ contains a Eulerian trail.

Theorem 19. Let I be an ideal of a ring $\mathcal{R}$ that is not prime such that $|I|$ is even, $|X|$ is even, and $|Y|$ is odd. Then, we have the following case.

(a) $T_{I}(\Gamma(\mathcal{R}))$ is Eulerian if and only if $\Gamma_{I}(\mathcal{R})$ is Eulerian.

(b) $T_{I}\left(\Gamma(\mathcal{R})\right.$ ) contains a Eulerian trail if and only if $\Gamma_{I}(\mathcal{R})$ contains a Eulerian trail.

Remark 10. In view of Theorems 16,18 , and 19 , since $|I|$ is even and $|\mathcal{R}|$ is odd, there is no graph on $n$ vertices that can be realized as $T_{I}(\Gamma(\mathcal{R}))$ for some ring $\mathcal{R}$ and an ideal I of $\mathcal{R}$.

Theorem 20. Let I be a non-prime ideal of a ring $\mathcal{R}$ such that $|I|=2,|X|$ is even, and $|Y|$ is even. Then, $T_{I}\left(\Gamma(\mathcal{R})\right.$ ) contains a Eulerian trail if and only if $\Gamma_{I}(\mathcal{R})$ is Eulerian.

Proof. Suppose that $T_{I}(\Gamma(\mathcal{R}))$ contains a Eulerian trail. Then, each vertex of $T_{I}(\Gamma(\mathcal{R}))$ has an even degree except for two vertices that have odd degrees. Since $I$ is not a prime ideal of $\mathcal{R}$, the vertex set of $T_{I}(\Gamma(\mathcal{R}))$ consists of $I, X$, and $Y$. Let $u$ and $v$ be the two vertices of odd degree and let $w_{1}, w_{2}, \ldots, w_{n}$ be the vertices of even degree. Since $|X|$ and $|Y|$ are even and $|I|=2, u, v \in I$. If we assume that at least one of $u, v \in X$, then $|I|$ is odd, which is a contradiction, and if we assume that at least one of $u, v \in Y$, then we have more than two odd vertices in $T_{I}(\Gamma(\mathcal{R}))$. Therefore, $T_{I}(\Gamma(\mathcal{R}))$ has no Eulerian trail, which is a contradiction. Thus, the two elements of $I$ are the only ones that are odd and all other elements of $X$ and $Y$ are even. Now, we know that $\Gamma_{I}(\mathcal{R})$ is a subgraph of $T_{I}(\Gamma(\mathcal{R})$ ), i.e., the set $Y$ is the set of vertex of $\Gamma_{I}(\mathcal{R})$, but to obtain the exact number of edges that incident on each vertex of $\Gamma_{I}(\mathcal{R})$, we remove the edges join all vertex of $I$ with each vertex of $Y$. Thus, the degree of each vertex of $\Gamma_{I}(\mathcal{R})$ is same as the degree of each vertex of $Y$ in $T_{I}(\Gamma(\mathcal{R}))$ subtract $|I|$. Therefore, the degree of each vertex of $\Gamma_{I}(\mathcal{R})$ is even. Hence, $\Gamma_{I}(\mathcal{R})$ is Eulerian.

Conversely, suppose that $\Gamma_{I}(\mathcal{R})$ is Eulerian. Then, each vertex of $\Gamma_{I}(\mathcal{R})$ has an even degree. Thus, each vertex of $Y$ in $\Gamma_{I}(\mathcal{R})$ has an even degree. Now, we will construct $T_{I}(\Gamma(\mathcal{R}))$. First, we have two vertices of the set $I$ adjacent to all vertex of $\mathcal{R}$, i.e., adjacent to $(|I|-1+|X|+|Y|)$ vertices. Since $|I|=2,|X|$ and $|Y|$ are even, and the degree of each vertex of $I$ is odd, i.e., we have two vertices of $I$ being odd. Now, if we have an even vertex of set $X$, which is adjacent to all vertices of $I$ only. Thus, the degree of each vertex of $X$ is even. Finally, we have an even vertex of set $Y$ adjacent to all vertex of $I$ and at least one vertex of $Y$. Since $|I|=2$ is even and each vertex of $Y$ in $\Gamma_{I}(\mathcal{R})$ has an even degree, the degree of each vertex of $Y$ in $T_{I}(\Gamma(\mathcal{R}))$ is even. Thus, each vertex of $T_{I}(\Gamma(\mathcal{R}))$ has an even degree except for two vertices, which have odd degrees. Hence, $T_{I}(\Gamma(\mathcal{R}))$ contains a Eulerian trail.

Theorem 21. Let I be an ideal of a ring $\mathcal{R}$ that is not prime such that $|I|=2,|X|$ is odd, and $|Y|$ is odd. Then, $T_{I}(\Gamma(\mathcal{R}))$ contains a Eulerian trail if and only if $\Gamma_{I}(\mathcal{R})$ is Eulerian.

Proof. By the same arguments used in the above Theorem 18, the proof is clear.

Example 3. Let $\mathcal{R}=\mathbb{Z}_{12}$ and $I=(6)=\{0,6\}$. Then, $Y=\{2,3,4,8,9,10\}$ and $X=$ $\{1,5,7,11\}$. We observe that $\Gamma_{I}(\mathcal{R})$ is Eulerian and $T_{I}(\Gamma(\mathcal{R}))$ contains a Eulerian trail (see Figure 9). 

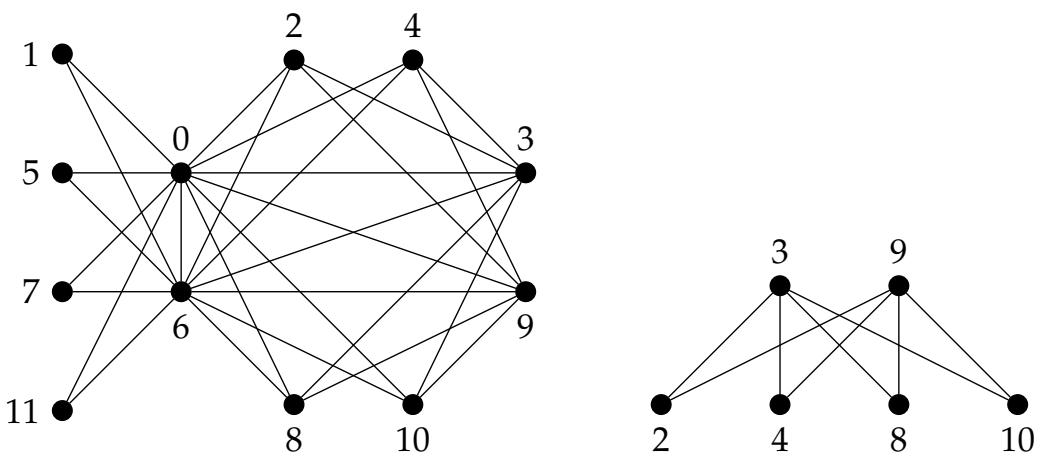

Figure 9. (left) $T_{I}(\Gamma(\mathcal{R}))$ and (right) $\Gamma_{I}(\mathcal{R})$, when $\mathcal{R}=\mathbb{Z}_{12}$ and $I=(6)$.

Theorem 22. Let I be an ideal of a ring $\mathcal{R}$ such that $|\mathcal{R}|=n \geq 3$. If $I=\mathcal{R}$, then $T_{I}(\Gamma(\mathcal{R}))$ is Hamiltonian.

Proof. Suppose that $I=\mathcal{R}$. Then, by Corollary $5, T_{I}(\Gamma(\mathcal{R}))$ is a complete graph. Hence, $T_{I}(\Gamma(\mathcal{R}))$ is Hamiltonian.

Theorem 23. Let I be a zero ideal of a ring $\mathcal{R}$. Then, $T_{I}(\Gamma(\mathcal{R}))$ cannot be Hamiltonian.

Proof. Suppose that $I=(0)$. Then, by Corollary $9, T_{I}(\Gamma(\mathcal{R}))$ has a cut-vertex. Hence $T_{I}(\Gamma(\mathcal{R}))$ cannot be Hamiltonian.

Theorem 24. Let I be a nonzero ideal of a ring $\mathcal{R}$ such that $|\mathcal{R}|=n \geq 3$. If $|I| \geq \frac{n}{2}$, then $T_{I}(\Gamma(\mathcal{R}))$ is Hamiltonian.

Proof. Suppose that $u$ and $v$ are any two vertices of $T_{I}(\Gamma(\mathcal{R}))$. Then, we have the following cases.

Case $(i)$ : If $u$ and $v$ are adjacent for all $u, v \in T_{I}(\Gamma(\mathcal{R}))$, then $T_{I}(\Gamma(\mathcal{R}))$ is complete. Therefore, $T_{I}(\Gamma(\mathcal{R}))$ is Hamiltonian.

Case $(i i)$ : If $u$ and $v$ are nonadjacent for some $u, v \in T_{I}(\Gamma(\mathcal{R}))$, then by Remarks 1 (viii), (ix), and $(x)$, we have the following subcases:

Subcase $(a)$ : If $u, v \in X$, then by Corollary $7, \operatorname{deg}(u)=\operatorname{deg}(v)=|I|$. Thus, $\operatorname{deg}(u)+$ $\operatorname{deg}(v)=|I|+|I| \geq \frac{n}{2}+\frac{n}{2}=n$. Hence, $T_{I}(\Gamma(\mathcal{R}))$ is Hamiltonian.

Subcase $(b)$ : If $u, v \in Y$, then by Corollary $7, \operatorname{deg}(u)=\operatorname{deg}(v) \geq|I|$. Thus, $\operatorname{deg}(u)+$ $\operatorname{deg}(v) \geq|I|+|I| \geq \frac{n}{2}+\frac{n}{2}=n$. Hence, $T_{I}(\Gamma(\mathcal{R}))$ is Hamiltonian.

Subcase (c): If $u \in X$ and $v \in Y$, then by Corollary $7, \operatorname{deg}(u)=|I|$ and $\operatorname{deg}(v) \geq|I|$. Thus, $\operatorname{deg}(u)+\operatorname{deg}(v) \geq|I|+|I| \geq \frac{n}{2}+\frac{n}{2}=n$. Hence, $T_{I}(\Gamma(\mathcal{R}))$ is Hamiltonian.

Corollary 13. Let I be a nonzero ideal of a ring $\mathcal{R}$ such that $|\mathcal{R}|=n \geq 3$. If $|I| \geq \frac{n}{2}$ for each pair $u, v$ of $X$, then $T_{I}(\Gamma(\mathcal{R}))+u v$ is Hamiltonian if and only if $T_{I}(\Gamma(\mathcal{R}))$ is Hamiltonian.

Corollary 14. Let I be a nonzero ideal of a ring $\mathcal{R}$. Then, I is a maximal ideal of a ring $\mathcal{R}$ if and only if $T_{I}(\Gamma(\mathcal{R}))$ is Hamiltonian.

\section{Conclusions}

We considered a generalization of dot total graph of $\mathcal{R}$ as well as an ideal-based zero-divisor graph. We showed that $T_{I}(\Gamma(\mathcal{R}))$ is connected and has a small diameter of at most two. Furthermore, we studied the connectivity, clique number and the girth of $T_{I}(\Gamma(\mathcal{R}))$. In addition, the cases when $T_{I}(\Gamma(\mathcal{R}))$ is Eulerian, Hamiltonian, and $T_{I}(\Gamma(\mathcal{R}))$ contains a Eulerian trail. For future work, the application of this graph to the study on Laplacian eigenvalues of an ideal-based dot total graph, which is closely related to the work in the paper [6], can be investigated. Additionally, the energy of an ideal-based dot total graph, which is related to the recent work in $[5,7]$, requires more consideration. The 
purpose of studying this type of graph is beneficial from its application point of view in practical life, such as networks, especially communication networks, which will be studied in an independent manuscript.

Author Contributions: Investigation, M.A., J.H.A., A.M.A. and A.A.; Writing-original draft, M.A., J.H.A., A.M.A. and A.A.; Writing—review and editing, M.A., J.H.A., A.M.A. and A.A. All authors have read and agreed to the published version of the manuscript.

Funding: This research received no external funding.

Institutional Review Board Statement: Not applicable.

Informed Consent Statement: Not applicable.

Data Availability Statement: Not applicable.

Acknowledgments: The authors are grateful to the referees for their valuable comments and suggestions.

Conflicts of Interest: The authors declare no conflict of interest.

\section{References}

1. Mohammad, A.; Asalool, J.H.; Mohit, K. The total graph of commutative ring with respect to multiplication. Italian J. Pure Appl. Math. 2022, in press.

2. Redmond, S.P. An ideal-based zero-divisor graph of a commutative ring. Comm. Algebra 2003, 31, 4425-4443. [CrossRef]

3. Anderson, D.F.; Livingston, P.S. The zero-divisor graph of a commutative ring. J. Algebra 1999, 217, 434-447. [CrossRef]

4. Beck, I. Coloring of commutative rings. J. Algebra 1988, 116, 208-226. [CrossRef]

5. Nath, R.K.; Fasfous, W.N.T.; Das, K.C.; Shang, Y. Common Neighborhood Energy of Commuting Graphs of Finite Groups. Symmetry 2021, 13, 1651. [CrossRef]

6. Rather, B.A.; Pirzada, S.; Naikoo, T.A.; Shang, Y. On Laplacian eigenvalues of the zero-divisor graph associated to the ring of integers modulo n. Mathematics 2021, 9, 482. [CrossRef]

7. Shang, Y. A note on the commutativity of prime near-rings. Algebra Colloq. 2015, 22, 361-366. [CrossRef]

8. Diestel, R. Graph Theory; Springer: New York, NY, USA, 1997. 